\title{
Space-Time Adaptive Processing in Bistatic Passive Radar Exploiting Group Sparsity
}

\author{
Qisong $\mathrm{Wu}^{\dagger}$, Yimin D. Zhang ${ }^{\dagger}$, Moeness G. Amin ${ }^{\dagger}$, and Braham Himed ${ }^{\ddagger}$ \\ $\dagger$ Center for Advanced Communications, Villanova University, Villanova, PA 19085, USA \\ $\ddagger$ RF Technology Branch, Air Force Research Lab (AFRL/RYMD), WPAFB, OH 45433, USA
}

\begin{abstract}
In this paper, we propose a novel method to estimate the clutter covariance matrix (CCM) and perform space-time adaptive processing (STAP) for effective clutter suppression based on a small number of secondary data samples. By exploiting the group sparsity of the angle-Doppler domain clutter profile shared by nearby range cells in a bistatic passive radar platform, we first apply the complex multi-task Bayesian compressive sensing (CMT-BCS) algorithm to reconstruct the sparse clutter profile based on the secondary data samples. The clutter profile in the range cell under test is then obtained within the common clutter support over all secondary data samples to ensure the exclusion of target signals in the estimated CCM. Compared to the conventional STAP method, the number of required secondary samples is significantly reduced due to the group sparsity of the clutter profile. The effectiveness of the proposed algorithm is verified using simulation results.
\end{abstract}

\section{INTRODUCTION}

Passive radar (PR) systems utilize broadcast, navigation, and communication signals as sources of opportunity to perform various surveillance tasks. PR systems have attracted significant interests due to their distinct advantages, such as low cost and covertness, as compared to conventional active radar systems [1], [2].

Airborne PR systems suffer from strong ground clutter that may obscure weak target signals. Space-time adaptive processing (STAP) is an effective technique for detecting slowly moving targets in a cluttered scene. Conventional STAP techniques require a large number of secondary data samples to yield a reliable estimation of the clutter covariance matrix (CCM) [3]. A significant amount of work, such as reduced-rank STAP, has been performed to relax the restriction of the number of secondary data samples [4]. In PR systems, however, a sufficient number of secondary data samples may not be available to construct the CCM with a sufficient rank, because narrowband signals that correspond to a poor range resolution are used.

More recently, new STAP techniques have been developed based on the latest advances in the area of sparse signal reconstruction and compressive sensing (CS). It has been shown that, by taking advantages of the intrinsic sparsity of the clutter profile in the angleDoppler domain, the clutter spectrum can be recovered using very few data samples [5]-[8]. In these approaches, the sparse clutter profile estimation is cast as a regularized optimization problem which can be readily solved using available CS methods. The estimated clutter profile is then used to construct the CCM to achieve effective clutter suppression. In [5] and [8], a single data sample under test is used to estimate the scatterer profiles. In [6] and [7], two approaches are proposed for the clutter profile estimation by utilizing multiple data samples. One such approach is to average the estimated profiles, which are individually estimated based on their own range cell, to represent the clutter profile in the range cell under test. Another method is to choose the maximum values from multiple separately estimated clutter profiles as the profile under test.

The work of Q. Wu, Y. D. Zhang, and M. G. Amin was supported in part by a subcontract with Defense Engineering Corporation for research sponsored by the Air Force Research Laboratory under Contract FA8650-12-D-1376.
There are several critical issues involved with these techniques. First, to avoid suppression of the target signals during the STAP procedure, the target signals in the range cell under test must be excluded in the reconstructed clutter profiles. In these approaches, this requirement is satisfied by assuming that target signals are weak and, thereby, are absent from the reconstructed clutter profile. It is, however, difficult to guarantee such an outcome in different situations. Second, preserving the high fidelity of the clutter spectrum requires a high-resolution clutter profile estimation in the angle-Doppler domain, which may yield a high coherence in the measurement (dictionary) matrix. The performance of most existing CS methods degrades significantly when the dictionary matrix becomes highly coherent.

In this paper, we propose a novel approach to accurately estimate the CCM and implement STAP based on a very small number of secondary data samples. By exploiting the group sparsity of the clutter, i.e., nearby range cells share the same non-zero clutter support in the angle-Doppler domain but their values are different in general, we first use the secondary data samples to estimate this clutter-only support. The actual clutter profile in the range cell under test is then estimated within the clutter-only support and, as such, ensures the exclusion of the target signals in the estimated CCM. The complex multi-task Bayesian compressive sensing (CMT-BCS) [9] algorithm is exploited to perform high-resolution estimation of the angleDoppler domain clutter profile because Bayesian-based CS methods are insensitive to the dictionary matrix coherence. The proposed method does not require the independent and identically distributed (i.i.d.) condition of the secondary data samples as typically assumed in conventional STAP. The effectiveness of the proposed algorithm is verified using simulation results.

Notations: We use lower-case (upper-case) bold characters to denote vectors (matrices). In particular, $\mathbf{I}_{N}$ denotes the $N \times N$ identity matrix. (.) ${ }^{*}$ denotes complex conjugate, whereas $(.)^{T}$ and $(.)^{H}$, respectively, denote the transpose and conjugate transpose of a matrix or vector. $\operatorname{diag}(\mathbf{x})$ represents a diagonal matrix that uses the elements of $\mathbf{x}$ as its diagonal elements. $\|\cdot\|_{2}^{2}$ implies the Euclidean $\left(l_{2}\right)$ norm of a vector. $\operatorname{Tr}($.$) denotes the trace of a matrix. \mathrm{P}_{\mathrm{r}}(\cdot)$ expresses the probability density function (pdf), and $\mathcal{N}(x \mid a, b)$ denotes the random variable $x$ follows a Gaussian distribution with mean $a$ and variance $b . \operatorname{In}$ addition, $\operatorname{Re}(x)$ and $\operatorname{Im}(x)$ are the real and imaginary parts of complex element $x$, respectively.

\section{System And Signal Model}

Consider a bistatic PR system consisting of a stationary transmitters and a moving receiver as depicted in Fig. 1. The transmitter is assumed to be located at a stationary and known position $\mathbf{p}_{\mathrm{T}}=$ $\left[p_{\mathrm{T}, x}, p_{\mathrm{T}, y}, p_{\mathrm{T}, z}\right]^{T}$. The radar receiver, which utilizes an $N$-element uniform linear array (ULA) with inter-element spacing $d$, moves in a straight line with a constant velocity $v_{R}$ in the $x$-direction. The initial position of the reference sensor is assumed to be $\mathbf{p}_{\mathrm{R}}(0)=$ 


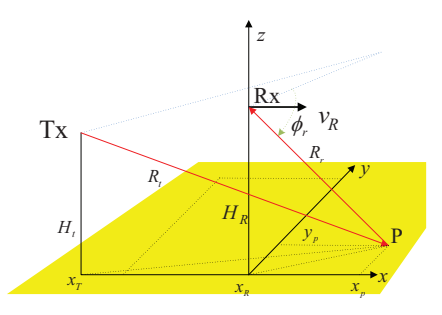

Fig. 1. Bistatic passive radar geometry.

$\left[p_{\mathrm{R}, x}(0), 0, H_{\mathrm{R}}\right]^{T}$. As such, the position of the reference sensor at time instant $t$ is expressed as $\mathbf{p}_{\mathrm{R}}(t)=\left[p_{\mathrm{R}, x}(0)+v_{\mathrm{R}} t, 0, H_{\mathrm{R}}\right]^{T}$.

The signal vector observed at the receive array is expressed as,

$$
\mathbf{x}(t)=\mathbf{x}_{c}(t)+\mathbf{x}_{s}(t)+\mathbf{n}(t),
$$

where $\mathbf{x}_{c}(t)$ denotes the clutter vector and $\mathbf{x}_{s}(t)$ represents the target signal vector. In addition, the additive noise vector $\mathbf{n}(t)$ is characterized as i.i.d. complex Gaussian with zero mean. The clutter and the target signal are described in the next two sub-sections. Note that the direct-path interference from the transmitter is not considered here as it can be effectively suppressed in the received data by exploiting interference cancellation techniques [10].

\section{A. Clutter}

The clutter observed at the receiver is modeled as a summation of $N_{c}$ discrete and statistically independent scatterers, given by,

$$
\begin{aligned}
\mathbf{x}_{c}(t) & =\sum_{m=1}^{N_{c}} \frac{\sqrt{P_{\mathrm{T}}} G_{m} \sigma_{c, m}}{r_{\mathrm{TC}, m} r_{\mathcal{C R}, m}(t)} \mathbf{a}\left(\phi_{m}\right) \\
& \cdot s\left[t-\tau_{\mathrm{TC}, m}(t)-\tau_{\mathcal{C} \mathrm{R}, m}(t)\right] e^{-j 2 \pi f_{\mathcal{C}}\left(\tau_{\mathrm{T} \mathcal{C}, m}+\tau_{\mathcal{C R}, m}(t)\right)},
\end{aligned}
$$

where $P_{\mathrm{T}}$ is the transmit power from the illuminator, and $G_{m}$ is the transmit antenna gain. $r_{\mathrm{T} C, m}, m \in 1, \cdots, N_{c}$, is the range from the transmitter and the $m$ th clutter scatterer, and $r_{\mathcal{C R}_{\mathrm{R}, m}}(t)$ is the range from the same scatterer to the receiver at time instant $t$. The delays corresponding to $r_{\mathrm{TC}, m}$ and $r_{\mathcal{C R}, m}(t)$ are respectively denoted as $\tau_{\mathrm{TC}, m}$ and $\tau_{\mathcal{C R}, m}(t)$. In addition, $\sigma_{c, m}$ is the reflectivity of the $m$ th clutter scatterer, and $s(t)$ is the emitted signal waveform. Furthermore,

$$
\mathbf{a}\left(\phi_{m}\right)=\left[1, e^{j \kappa d \sin \left(\phi_{m}\right)}, \ldots, e^{j \kappa(N-1) d \sin \left(\phi_{m}\right)}\right]^{T} \in \mathcal{C}^{N}
$$

is the steering vector of the receive array toward the direction of the $m$ th clutter scatterer with a direction-of-arrival (DOA) $\phi_{m}$, which is defined as the cone angle with respect to the $x$-axis. In the above expressions, $\kappa=2 \pi / \lambda$ is the wavenumber, $\lambda=c / f_{c}$ is the wavelength of the emitted signal, $c$ is the velocity of light, and $f_{c}$ is the carrier frequency.

In practice, the waveform emitted from the illuminator is available at the receiver from, e.g., the use of reference channels [11]. Performing matched filtering and the $n$th range bin yields the output of the clutter signal at azimuth time $t_{l}=l T, l=0, \ldots, L-1$. Assuming a small value of $T$, then the output signal is expressed as

$$
\mathbf{y}_{c}^{(n)}\left(t_{l}\right)=\sum_{m=1}^{N_{c}} \frac{\sqrt{P_{\mathrm{T}}} G_{m} \sigma_{c, m}}{r_{\mathrm{T},{ }_{m}} r_{\mathcal{C}, m}(t)} \rho \mathbf{a}\left(\phi_{m}\right) e^{j 2 \pi \nu_{m}\left(t_{l}-(L-1) T / 2\right)},
$$

where $\rho=\rho(0)$, with

$$
\rho_{i}(\tau)=\int_{t_{l}}^{t_{l}+T} s(t) s^{*}(t+\tau) d t
$$

denoting the signal auto-correlation function, which is independent of $t_{l}$ as most waveforms have a stable auto-correlation property [2].
In addition,

$$
\nu_{m}=-\frac{1}{\lambda} \frac{d}{d t} r_{m \mathrm{R}}(t)=-\frac{1}{\lambda} v_{\mathrm{R}} \cos \phi_{m}
$$

is the Doppler frequency of the $m$ th clutter scatterer and is determined by the scatterer-receiver path. It should be noted in Eq. (6) that the clutter Doppler frequency only depends on the receiver velocity and is independent of range cells. This characteristic invites the use of group sparsity of the clutter profiles among nearby range cells to obtain the common clutter support in the bistatic configuration.

Stacking $\mathbf{y}_{c}^{(n)}\left(t_{l}\right)$ over the $L$ collected azimuth time samples yields

$$
\tilde{\mathbf{y}}_{c}=\sum_{m=1}^{N_{c}} \frac{\sqrt{P_{\mathrm{T}}} G_{m} \sigma_{c, m}}{r_{\mathrm{TC}, m} r_{\mathcal{C R}, m}(t)} \rho \mathbf{h}\left(\nu_{m}, \phi_{m}\right)
$$

where

$$
\mathbf{h}\left(\nu_{m}, \phi_{m}\right)=\mathbf{b}\left(\nu_{m}\right) \otimes \mathbf{a}\left(\phi_{m}\right) \in \mathcal{C}^{N L}
$$

is the spatio-temporal signature of the $m$ th scatterer, and

$$
\mathbf{b}\left(\nu_{m}\right)=\left[e^{-j 2 \pi \nu_{m}(L-1) T / 2}, \ldots, e^{j 2 \pi \nu_{m}(L-1) T / 2}\right]^{T} \in \mathcal{C}^{L}
$$

is the temporal signature vector of the $m$ th clutter scatterer.

\section{B. Desired Signal}

Assume that $Q$ moving targets, all located on ground within an area, are inseparable via the bistatic ranges due to the coarse range resolution in the $\mathrm{PR}$ system. The $q$ th ground moving target is located at $\mathbf{p}_{q}=\left[x_{q}, y_{q}, 0\right]^{T}$ with a velocity of $\mathbf{v}_{q}=\left[v_{x q}, v_{y q}, 0\right]^{T}$. The desired signal $\mathbf{x}_{s}(t)$ received at the receiver is expressed as

$$
\begin{aligned}
\mathbf{x}_{s}(t) & =\sum_{q=1}^{Q} \frac{\sqrt{P_{\mathrm{T}}} G_{q} \sigma_{q}}{r_{\mathrm{T} q} r_{q \mathrm{R}}} \mathbf{a}\left(\phi_{q}\right) \\
& \cdot s\left[t-\tau_{\mathrm{T}_{q}}(t)-\tau_{q \mathrm{R}}(t)\right] e^{-j 2 \pi f_{c}\left[\tau_{\mathrm{T}_{q}}(t)+\tau_{q \mathrm{R}}(t)\right]},
\end{aligned}
$$

where $\sigma_{q}$ is the complex reflection coefficient associated with the radar cross section (RCS) of the $q$ th target. In addition, $\tau_{\mathrm{T} q}(t)$ and $\tau_{q \mathrm{R}}(t)$ are delays respectively corresponding to the range $r_{\mathrm{T} q}(t)$ between the transmitter and the $q$ th moving target, and the range $r_{q \mathrm{R}}(t)$ between the $q$ th moving target and the receiver.

In a similar manner with the clutter, by performing matched filtering at a given area, specified by delay $\tau_{n}$, we obtain the matched filter output at azimuth time $t_{l}=l T, l=0, \ldots, L-1$, as

$$
\mathbf{y}_{s}^{(n)}\left(t_{l}\right)=\sum_{q=1}^{Q} \frac{\sqrt{P_{\mathrm{T}}} G_{q} \sigma_{q}}{r_{\mathrm{T} q} r_{q \mathrm{R}}} \rho \mathbf{a}\left(\phi_{q}\right) e^{j 2 \pi \nu_{q}\left[t_{l}-(L-1) T / 2\right]},
$$

where $\nu_{q}$ denotes Doppler frequency of the $q$ th moving target and is determined by the change rate of the combined bistatic range, which can be expressed by

$$
\begin{aligned}
\nu_{q}= & -\frac{1}{\lambda} \cdot \frac{d}{d t}\left[r_{\mathrm{T} q}(t)+r_{q \mathrm{R}}(t)\right] \\
= & -\frac{\left[\mathbf{p}_{\mathrm{T}}-\mathbf{p}_{q}(0)\right]^{T} \cdot \mathbf{v}_{q}}{\lambda\left\|\mathbf{p}_{\mathrm{T}}-\mathbf{p}_{q}(0)\right\|}-\frac{\left[\mathbf{p}_{\mathrm{R}}(0)-\mathbf{p}_{q}(0)\right]^{T} \cdot \mathbf{v}_{q}}{\lambda\left\|\mathbf{p}_{\mathrm{R}}(0)-\mathbf{p}_{q}(0)\right\|} \\
& -\frac{\left[\mathbf{p}_{\mathrm{R}}(0)-\mathbf{p}_{q}(0)\right]^{T} \cdot \mathbf{v}_{R}}{\lambda\left\|\mathbf{p}_{\mathrm{R}}(0)-\mathbf{p}_{q}(0)\right\|},
\end{aligned}
$$

where $\mathbf{p}_{q}(0)$ is the the initial position of the $q$ th moving target, and $\mathbf{v}_{R}=\left[v_{R}, 0,0\right]^{T}$ is the velocity vector of the radar receiver. The Doppler frequency $\nu_{q}$ contains two components. The first component includes the first two terms on the right-hand side of Eq. (12) and represents the contribution of the moving target, whereas the second component is given as the third term on the right-hand side of Eq. (12) and represents the contribution of the moving receiver. 
Stacking $\mathbf{y}_{s}^{(n)}\left(t_{l}\right)$ over the $L$ collected azimuth time samples results in the following signal vector,

$$
\tilde{\mathbf{y}}_{s}=\sum_{q=1}^{Q} \frac{\sqrt{P_{\mathrm{T}}} G_{q} \sigma_{q}}{r_{\mathrm{T} q} r_{q \mathrm{R}}} \rho \mathbf{h}\left(\nu_{q}, \phi_{q}\right)
$$

where

$$
\mathbf{h}\left(\nu_{q}, \phi_{q}\right)=\mathbf{b}\left(\nu_{q}\right) \otimes \mathbf{a}\left(\phi_{q}\right) \in \mathcal{C}^{N L}
$$

is the spatio-temporal signature of the $q$ th target, where $\mathbf{a}\left(\phi_{q}\right)$ and $\mathbf{b}\left(\nu_{q}\right)$ are similarly defined as in Eq. (3) and Eq. (9).

\section{Clutter Profile Estimation and Clutter SUPPRESSION}

Because of the sparsity of the clutter in the angle-Doppler domain, CS-based approaches are effective in estimating the clutter profile, which is key to performing STAP [5]-[8]. The CMT-BCS [9] extends the real-valued multi-task CS algorithm [12] for the reconstruction of complex-valued sparse signals, and yields improved performance by sharing the same hyper-parameters between the real and imaginary components. The CMT-BCS has been successfully applied to reconstruct sparse complex-valued signals and achieve improved accuracy in various applications [13]-[15]. For convenience, this method is briefly summarized in the Appendix based on [9].

In this section, considering the fact that the non-zero support of clutter profiles is shared by nearby range cells, we first employ a small number of secondary samples to learn the common clutter support. The exact clutter profile in the range cell under test is then obtained through CMT-BCS by considering the clutter-only entries within the learned clutter support.

According to the clutter signal model described in Section II, the matched filter output of the received signal in the range cell under test is expressed for the $k$ th bistatic pair as

$$
\tilde{\mathbf{y}}^{(\mathrm{t})}=\tilde{\mathbf{y}}_{c}^{(\mathrm{t})}+\tilde{\mathbf{y}}_{s}^{(\mathrm{t})}+\tilde{\mathbf{y}}_{n}^{(\mathrm{t})}=\tilde{\boldsymbol{\Phi}}\left(\tilde{\mathbf{w}}_{c}^{(\mathrm{t})}+\tilde{\mathbf{w}}_{s}^{(\mathrm{t})}\right)+\tilde{\mathbf{y}}_{n}^{(\mathrm{t})},
$$

where the superscript $(\mathrm{t})$ is used to emphasize the range under test, $\tilde{\mathbf{w}}_{c}^{(\mathrm{t})}$ and $\tilde{\mathbf{w}}_{s}^{(\mathrm{t})}$ are, respectively, $N_{s} N_{d} \times 1$ vectorized clutter and signal entries in the angle-Doppler domain, with $N_{d}$ and $N_{s}$ being the numbers of Doppler and azimuth grids defined in the angleDoppler domain, respectively, $\tilde{\boldsymbol{\Phi}}=\left[\mathbf{h}\left(\nu_{1}, \phi_{1}\right), \cdots, \mathbf{h}\left(\nu_{N_{d}}, \phi_{N_{s}}\right)\right] \in$ $\mathcal{C}^{M L \times N_{d} N_{s}}$, and $\tilde{\mathbf{y}}_{n}^{(\mathrm{t})}$ denotes the noise component. On the other hand, the matched filter output of the received signals in the $n_{t}$ th secondary samples, where the described target signals are absent, is expressed as

$$
\tilde{\mathbf{y}}^{\left(n_{t}\right)}=\tilde{\mathbf{y}}_{c}^{\left(n_{t}\right)}+\tilde{\mathbf{y}}_{n}^{\left(n_{t}\right)} .
$$

Rewriting the clutter term in Eq. (16) as the product of a dense dictionary matrix $\tilde{\boldsymbol{\Phi}}$ and a sparse vector $\tilde{\mathbf{w}}_{c}^{\left(n_{t}\right)}$, we have

$$
\tilde{\mathbf{y}}^{\left(n_{t}\right)}=\tilde{\boldsymbol{\Phi}} \tilde{\mathbf{w}}_{c}^{\left(n_{t}\right)}+\tilde{\mathbf{y}}_{n}^{\left(n_{t}\right)},
$$

where $\tilde{\mathbf{w}}_{c}^{\left(n_{t}\right)} \in \mathcal{C}^{N_{d} N_{s}}$.

In this paper, we consider a small number of secondary data samples from range cells that are close to that under test, and thus it is well justified that they share the same non-zero clutter support with that under test, i.e., their scatterers are located in the same positions in the angle-Doppler domain. However, the exact values of the scatterers generally differ for each range due to the random nature of the clutter. Note that the assumption of the common support is much more relaxed and practical compared to the i.i.d. requirement and, as we will show, we need much less number of secondary samples.

The sparse clutter profile, $\tilde{\mathbf{w}}_{c}^{\left(n_{t}\right)}$, can be effectively recovered based on Eq. (17) from secondary samples by exploiting the CMT-BCS algorithm. From this estimated common clutter support, we then form the clutter-only dictionary $\tilde{\boldsymbol{\Phi}}_{c s}$ as a sub-matrix of $\tilde{\boldsymbol{\Phi}}$ by extracting the columns whose corresponding coefficients in $\tilde{\mathbf{w}}_{c}^{\left(n_{t}\right)}$ have nonzero support. One way to confine the clutter estimate for the range cell under test is to project the received signal to the clutter profile through the least square (LS) algorithm, expressed as

$$
\hat{\mathbf{w}}_{c}^{(\mathrm{t})}=\left(\tilde{\boldsymbol{\Phi}}_{c s}^{H} \tilde{\boldsymbol{\Phi}}_{c s}\right)^{-1} \tilde{\boldsymbol{\Phi}}_{c s}^{H} \tilde{\mathbf{y}}^{(\mathrm{t})} .
$$

However, this approach does not yield a robust solution because of the fact that the estimated clutter coefficients based on the LS algorithm are sensitive to the perturbation of the noise, particularly when the columns of the measurement matrix $\tilde{\boldsymbol{\Phi}}_{c s}$ are highly coherent as a result of high-resolution clutter profile estimation. A more reliable and accurate solution to estimate the clutter profile is by solving the following equation based on the CMT-BCS algorithm,

$$
\tilde{\mathbf{y}}^{(\mathrm{t})}=\tilde{\boldsymbol{\Phi}}_{c s} \mathbf{w}_{c}^{(\mathrm{t})}+\tilde{\mathbf{y}}_{n}^{(\mathrm{t})} .
$$

Note that, when compared with Eq. (15), the measurement matrix $\tilde{\boldsymbol{\Phi}}$ is replaced by the clutter-only dictionary matrix $\tilde{\boldsymbol{\Phi}}_{c s}$ and $\mathbf{w}_{c}^{(\mathrm{t})}$ is the corresponding sub-vector. As such, the target signal term is avoided in the estimated clutter entries $\mathbf{w}_{c}^{(t)}$, in Eq. (19) because it is out of the clutter support obtained from the nearby range cells.

Once the clutter coefficient vector $\hat{\mathbf{w}}_{c}^{(t)}$ is estimated, the corresponding CCM is expressed as [5], [7]

$$
\hat{\mathbf{R}}_{\mathbf{z}}=\sum_{m=1}^{M}\left|\hat{\mathbf{w}}_{c}^{(\mathrm{t})}\left(\nu_{m}, \phi_{m}\right)\right|^{2} \mathbf{h}\left(\nu_{m}, \phi_{m}\right) \mathbf{h}^{H}\left(\nu_{m}, \phi_{m}\right)+\hat{\beta}_{0}^{2} \mathbf{I}_{N L},
$$

where $\hat{\beta}_{0}^{2}$ is the noise power and can be adaptively estimated from observation data as described in Eq. (30).

Based on the estimated CCM from Eq. (20), the weight vector for that range under test is obtained as [3],

$$
\mathbf{u}=\frac{\hat{\mathbf{R}}_{\mathbf{z}}^{-1} \mathbf{h}_{0}}{\mathbf{h}_{0}^{H} \hat{\mathbf{R}}_{\mathbf{z}}^{-1} \mathbf{h}_{0}},
$$

where $\mathbf{h}_{0}$ is the spatio-temporal signature of the hypothetic target.

\section{Simulation Results}

In the simulated bistatic PR system, a stationary transmitter is located $8 \mathrm{~km}$ away from the scene center, emitting DVB-T signals with the carrier frequency of $800 \mathrm{MHz}$. The initial position of the receiver is $[0,6,8]^{T} \mathrm{~km}$, and the receiver is equipped with a 20element uniform linear array (ULA) with an inter-element spacing of half of the minimum wavelength. The velocity of the receiver is $v_{\mathrm{R}}=[100,0,0]^{T} \mathrm{~m} / \mathrm{s}$. The range of Doppler frequency bandwidth is from $-300 \mathrm{~Hz}$ to $300 \mathrm{~Hz} .30$ azimuthal samples are used, yielding a total number of $N L=600$ spatio-temporal samples.

\section{A. Reconstruction of Clutter Profiles Based on CMT-BCS}

Fig. 2 depicts the clutter profiles with $n_{t}=4$ nearby secondary samples in the angle-Doppler domain. The angle-Doppler domain clutter profile is discretized into a grid of $N_{d}=90$ Doppler bins from $-300 \mathrm{~Hz}$ to $300 \mathrm{~Hz}$ and $N_{s}=40$ angle bins from $-180^{\circ}$ to $180^{\circ}$, yielding $K=3600$ entries. According to the guideline for sparse clutter profile recovery in STAP, it would be a desirable choice that the value of $N_{s} N_{d}$ ranges between $4 N L$ and $16 N L$ [16]. The clutter profiles in each angular bins are represented by two adjacent non-zero random complex entries, and thus the total number of nonzero entries is 80, as shown in Fig. 2. An additive Gaussian noise is added with a clutter-to-noise ratio (CNR) of $40 \mathrm{~dB}$.

To exhibit the advantages of CMT-BCS on the sparse signal reconstruction with group structure, we have tested several state-ofthe-art CS algorithms, including group Lasso (GLasso) [17], block orthogonal matching pursuit (BOMP) [18], for their performance of 

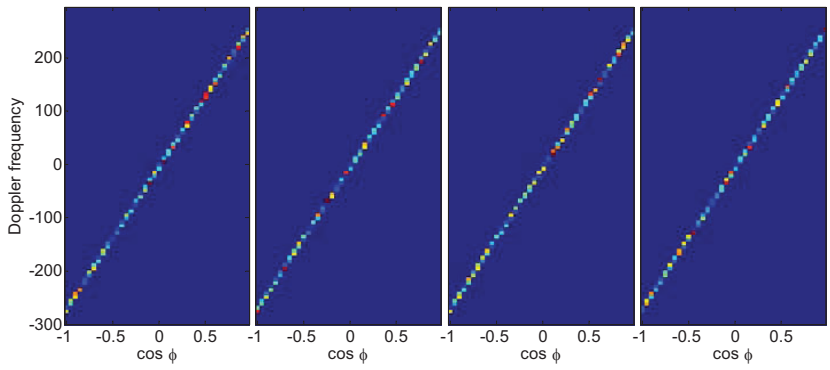

Fig. 2. Clutter profiles with 4 nearby secondary samples.

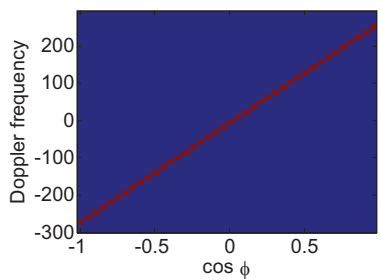

(a) Original profile

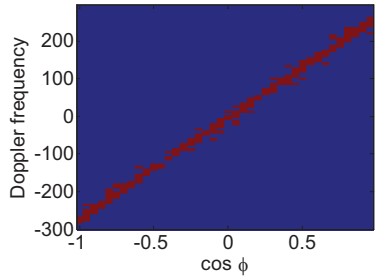

(c) GLasso

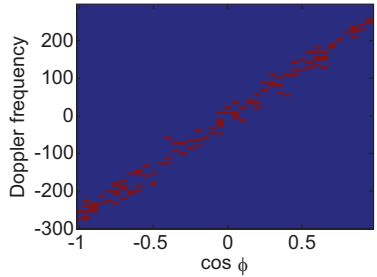

(b) BOMP

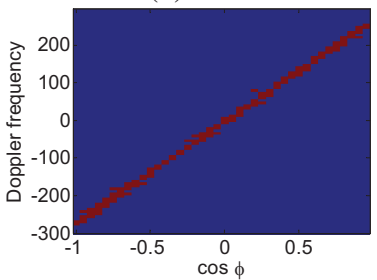

(d) CMT-BCS
Fig. 3. Estimated clutter support. (a) Original clutter support; estimated clutter support from (b) BOMP; (c) GLasso and (d) CMT-BCS.

clutter profile estimation. To quantitatively evaluate the performances of those algorithms, we introduce the normalized mean square error (NMSE), defined as $\left\|\hat{\boldsymbol{\vartheta}}-\boldsymbol{\vartheta}_{\text {gen }}\right\|^{2} /\left\|\boldsymbol{\vartheta}_{\text {gen }}\right\|^{2}$, as the metric, where $\hat{\boldsymbol{\vartheta}}$ is the estimate of the true scatterer coefficients $\boldsymbol{\vartheta}_{\text {gen }}$.

Fig. 3(a) shows the original clutter support. Fig. 3(b) shows the estimated support using the BOMP algorithm with the knowledge of the true sparsity assumed. This algorithm leads to a very poor clutter profile estimate and generates a high number of spurious clutter entries around the true positions because of the high coherence in the measurement matrix. The GLasso yields a better result when compared with the BOMP algorithm, resulting in a smaller NMSE. Despite its improved performance, this algorithm still has a high number of false clutter entries. Fig. 3(d) depicts the result based on the CMT-BCS with the group sparsity utilized. It is evident that the CMT-BCS algorithm reconstructs the non-zero clutter support with the least spurious positions and most concentrated structure among the methods being compared.

Once the clutter supports are acquired, we calculate the clutter scattering coefficients by utilizing both the LS algorithm in Eq. (18) and the CS algorithms in Eq. (19), respectively. Fig. 4(a) shows the true clutter profile. By utilizing the LS method in Eq. (18) based on the clutter support obtained from the CMT-BCS algorithm, we acquire the clutter profile shown in Fig. 4(b). For comparison, we also estimate the clutter profiles based on the clutter supports which are respectively obtained from the BOMP, GLasso, and CMT-BCS algorithms, and the results are depicted in Figs. 4(c)-4(e). In order to evaluate the accuracy of the estimated clutter profiles, we compare the NMSEs performance among those algorithms, as shown in Fig. 5(a). It is observed that the reconstruction error obtained from the LS algorithm is much larger than that from the CMT-BCS algorithm due

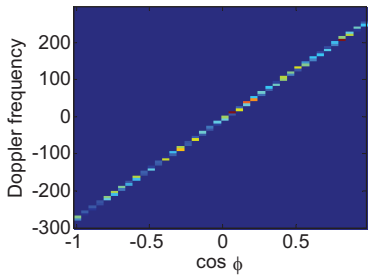

(a) Original profile

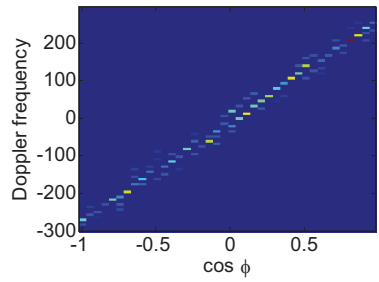

(c) BOMP

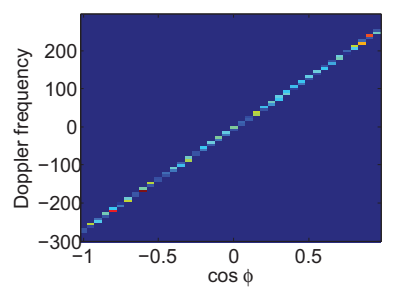

(b) LS

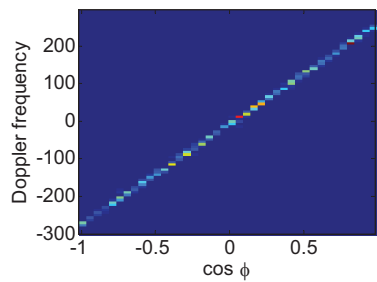

(d) GLasso

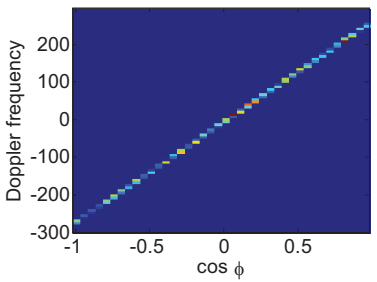

(e) CMT-BCS

Fig. 4. Estimated clutter profile in the range cell under test. (a) Original clutter profile; estimated clutter profile from (b) CMT-BCS with LS projection; (c) BOMP; (d) GLasso; and (e) CMT-BCS.

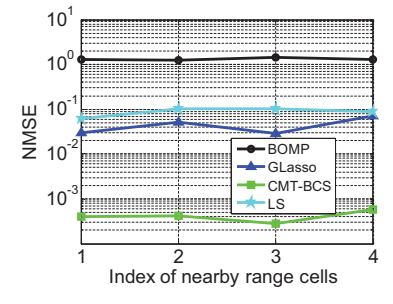

(a)

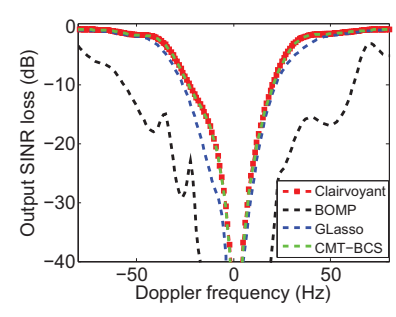

(b)
Fig. 5. Performance evaluation of different reconstruction algorithms. (a) NMSE. (b) Output SINR loss.

to its sensitivity to the noise and the measurement matrix coherency, even though both algorithms use the same clutter support.

To quantitatively evaluate the accuracy of the estimated CCMs in Eq. (20) based on the profiles obtained from the aforementioned CS algorithms, the output signal-inference-noise-ratio (SINR) loss, which is commonly used for the performance evaluation of STAP systems [3], is shown in Fig. 5(b). It is observed that the SINR loss in the CMT-BCS algorithm closely coincides with the Clairvoyant results because the CMT-BCS algorithm is capable of acquiring the best estimates of the clutter profile. On the other hand, the SINR loss based on the BOMP algorithm suffers the most due to poorest clutter profile estimation under the highly coherent measurement matrix.

\section{CONCLUSiON}

Conventional space-time adaptive processing (STAP) suffers from the requirement of a high number of independent identically distributed (i.i.d.) secondary data samples for a reliable estimation of the clutter covariance matrix (CCM). In this paper, a novel approach was proposed to estimate the CCM based on a very small number of secondary samples. Unlike traditional STAP technique which directly 
uses the secondary data for the CCM estimation, the secondary samples are used in this paper to estimate the common clutter support by exploiting the group sparsity of the clutter profile shared by nearby range cells. This estimated support is then used in the range cell under test to acquire the clutter profile with the target signal excluded. This strategy avoids the requirement of a high number of i.i.d. secondary samples, and avoid the serious shortcoming of existing CS-based STAP approaches which, by directly estimating the clutter profile from the data observed in the range cell under test, the moving target signals may be treated as clutter and subject to suppression.

\section{APPENDiX: Brief SuMmary of COMPlEX Multi-TASK BAYESIAN COMPRESSIVE SENSING [9]}

Consider a general multi-task CS model described as

$$
\check{\mathbf{y}}_{l}=\check{\mathbf{\Phi}}_{l} \check{\mathbf{w}}_{l}+\check{\varepsilon}_{l}, \quad l \in[1, \cdots, L],
$$

where each complex measurement $\check{\mathbf{y}}_{l} \in \mathcal{C}^{N_{l}}$ employs its own projection matrix $\check{\boldsymbol{\Phi}}_{l} \in \mathcal{C}^{N_{l} \times M}$, and $\check{\boldsymbol{\varepsilon}}_{l}$ denotes the additive complex noise vector. Decompose the $m$ th element $\breve{w}_{m l}$ of complex vector $\check{\mathbf{w}}_{l} \in \mathcal{C}^{M}$ into its real and imaginary components, denoted as $w_{m l}^{(\mathrm{R})}$ and $w_{m l}^{(\mathrm{I})}$, respectively, and form a real vector that consists of all real and imaginary components of complex vector $\breve{\mathbf{w}}_{l}$ as $\mathbf{w}_{l}=\left[w_{1 l}^{(\mathrm{R})}, \cdots, w_{M l}^{(\mathrm{R})}, w_{1 l}^{(\mathrm{I})}, \cdots, w_{M l}^{(\mathrm{I})}\right]^{T} \in \mathrm{R}^{2 M}$. By defining $\mathbf{y}_{l}=$ $\left[\operatorname{Re}\left(\check{\mathbf{y}}_{l}\right), \operatorname{Im}\left(\check{\mathbf{y}}_{l}\right)\right]^{T}$ and

$$
\boldsymbol{\Psi}_{l}=\left[\begin{array}{cc}
\operatorname{Re}\left(\check{\boldsymbol{\Phi}}_{l}\right) & -\operatorname{Im}\left(\check{\boldsymbol{\Phi}}_{l}\right) \\
\operatorname{Im}\left(\check{\boldsymbol{\Phi}}_{l}\right) & \operatorname{Re}\left(\check{\boldsymbol{\Phi}}_{l}\right)
\end{array}\right]
$$

Eq. (22) becomes

$$
\mathbf{y}_{l}=\Psi_{l} \mathbf{w}_{l}+\varepsilon_{l} .
$$

The prior distribution of real weight vector $\mathbf{w}_{l}$ that characterizes task $l$ is modeled as a zero-mean Gaussian distribution, which is shared between the real and imaginary components and across all $L$ groups, i.e.,

$$
\mathbf{w}_{l} \sim \mathcal{N}\left(\mathbf{w}_{l} \mid \mathbf{0}, \mathbf{A}\right)
$$

where

$$
\mathbf{A}=\operatorname{diag}\left(\alpha_{1}, \cdots, \alpha_{M}, \alpha_{M+1}, \cdots, \alpha_{2 M}\right),
$$

with $\alpha_{m}=\alpha_{m+M}$ denoting the variance of both $w_{m l}^{(\mathrm{R})}$ and $w_{m l}^{(\mathrm{I})}$, $m=1, \cdots, M \cdot w_{m l}^{(\mathrm{R})}$ and $w_{m l}^{(\mathrm{I})}$ tend to be zero with probability 1 for all $l \in[1, \ldots, L]$ when $\alpha_{m}$ is set to zero. By sharing the same parameter $\alpha_{m}$ for both real and imagery components, the CMT-BCS algorithm improves the sparsity of the estimated weight vectors and yields improved sparse signal recovery performance, when compared with the case when the real and imaginary components are independently treated.

When the parameters $\alpha$ and $\beta_{0}$ are specified, the posterior density function for $\mathbf{w}_{l}$ can be evaluated analytically based on Bayes' rule as

$$
\operatorname{Pr}\left(\mathbf{w}_{l} \mid \mathbf{y}_{l}, \boldsymbol{\Psi}_{l}, \boldsymbol{\alpha}, \beta_{0}\right)=\mathcal{N}\left(\mathbf{w}_{l} \mid \boldsymbol{\mu}_{l}, \boldsymbol{\Sigma}_{l}\right)
$$

where

$$
\boldsymbol{\mu}_{l}=\beta_{0}^{-1} \boldsymbol{\Sigma}_{l} \boldsymbol{\Psi}_{l}^{T} \mathbf{y}_{l}
$$

and

$$
\boldsymbol{\Sigma}_{l}=\left[\beta_{0}^{-1} \mathbf{\Psi}_{l}^{T} \mathbf{\Psi}_{l}+\mathbf{A}^{-1}\right]^{-1}
$$

respectively denote the mean and covariance of the scattering coefficients. A type-II maximum likelihood approximation employs a point estimate for $\beta_{0}$ and $\boldsymbol{\alpha}$ to maximize marginal likelihood, yielding,

$$
\begin{aligned}
& \alpha_{m}^{\text {(new) }}=\sqrt{\frac{\sum_{l=1}^{L}\left(\mathbf{w}_{m l}^{T} \mathbf{w}_{m l}+\mathbf{w}_{m+M, l}^{T} \mathbf{w}_{m+M, l}\right)}{\sum_{l=1}^{L} \operatorname{Tr}\left[\left(\mathbf{C}_{l}^{*}\right)^{-1}\left(\mathbf{\Psi}_{m l}^{T} \mathbf{\Psi}_{m l}+\mathbf{\Psi}_{m+M, l}^{T} \mathbf{\Psi}_{m+M, l}\right)\right]}}, \\
& \beta_{0}^{\text {(new) }}=\frac{1}{2 M L} \sum_{l=1}^{L} \operatorname{Tr}\left[\boldsymbol{\Sigma}_{l} \mathbf{\Psi}_{l} \mathbf{\Psi}_{l}^{T}\right]+\left\|\mathbf{y}_{l}-\mathbf{\Psi}_{l} \boldsymbol{\mu}_{l}\right\|_{2}^{2},
\end{aligned}
$$

where $\mathbf{C}_{l}=\beta_{0} \mathbf{I}+\mathbf{\Psi}_{l} \mathbf{A} \boldsymbol{\Psi}_{l}^{T} \in R^{2 N_{l} \times 2 N_{l}}$.

Note that $\alpha_{m}^{\text {(new) }}$ in Eq. (29) and $\beta_{0}^{\text {(new) }}$ in Eq. (30) are functions of $\boldsymbol{\mu}_{l}$ in Eq. (27) and $\boldsymbol{\Sigma}_{l}$ in Eq. (28), while $\boldsymbol{\mu}_{l}$ and $\boldsymbol{\Sigma}_{l}$ are functions of $\alpha_{m}$ and $\beta_{0}$. This suggests an iterative algorithm, which iterates between Eq. (27), Eq. (28) and Eq. (29), Eq. (30), until a convergence criterion is satisfied.

\section{REFERENCES}

[1] H. D. Griffths and C. J. Baker, "Passive coherent location radar systems. part 1: Performance prediction," IEE Proc. Radar, Sonar and Navig, vol. 152, no. 3, pp. 153-159, 2005.

[2] N. J. Willis and H. D. Griffiths (eds.), Advances in Bistatic Radar. SciTech, 2007.

[3] J. R. Guerci, Space-Time Adaptive Processing for Radar. Artech House, 2003.

[4] M. C. Wicks, M. Rangaswamy, R. Adve, and T. B. Hale, "Space-time adaptive processing: A knowledge-based perspective for airborne radar," IEEE Signal Proc. Mag., vol. 23, no. 1, pp. 51-65, 2006.

[5] I. W. Selesnick, S. U. Pillai, K. Y. Li, and B. Himed, "Single-sample STAP using sparsity," in Proc. U.S. Australia Joint Workshop on Defense Applications of Signal Processing, (Lihue, HI), Sept. 2009.

[6] K. Sun, H. Zhang, G. Li, H. Meng, and X. Wang, "Airborne radar STAP using sparse recovery of clutter spectrum," http://arxiv.org/pdf/1008.4185v1.

[7] K. Sun, H. Meng, Y. Wang, and X. Wang, "Direct data domain STAP using sparse representation of clutter spectrum," Signal Proc., vol. 91, no. 9, pp. 2222-2236, 2011.

[8] Y. D. Zhang and B. Himed, "Space-time adaptive processing in bistatic passive radar exploiting clutter sparsity," in Proc. IEEE Radar Conf., (Cincinati, OH), May 2014.

[9] Q. Wu, Y. D. Zhang, M. G. Amin, and B. Himed, "Complex multitask Bayesian compressive sensing," in Proc. IEEE ICASSP, (Florence, Italy), May 2014.

[10] D. Tan, M. Lesturgie, H. Sun, and Y. Lu, "Space-time interference analysis and suppression for airborne passive radar using transmissions of opportunity," IET Radar, Sonar and Navig., vol. 8, no. 2, pp. 142-152, 2014.

[11] C. R. Berger, B. Demissie, J. Heckenbach, P. Willett, and S. Zhou, "Signal processing for passive radar using OFDM waveforms," IEEE J. Sel. Topics Signal Proc., vol. 4, no. 1, pp. 226-238, 2010.

[12] S. Ji, D. Dunson, and L. Carin, "Multitask compressive sampling," IEEE Trans. Signal Proc., vol. 57, no. 1, pp. 92-106, 2009.

[13] Q. Wu, Y. D. Zhang, M. G. Amin, and B. Himed, "Multi-static passive radar SAR imaging based on Bayesian compressive sensing," in Proc. SPIE Defense, Security, and Sensing, (Baltimore, MD), May 2014.

[14] Q. Wu, Y. D. Zhang, M. G. Amin, and F. Ahmad, "Through-the-wall radar imaging based on modified bayesian compressive sensing," in Proc. IEEE China Summit and Int. Conf. Signal and Inform. Proc., (Xi'an, China), July 2014.

[15] Q. Wu, Y. D. Zhang, M. G. Amin, and B. Himed, "Multi-task Bayesian compressive sensing exploiting intra-task dependency," IEEE Signal Proc. Lett., vol. 22, no. 4, pp. 430-434, 2015.

[16] Z. C. Yang, Z. Liu, X. Li, and L. Nie, "Performance analysis of STAP algorithms based on fast sparse recovery techniques," Progress In Electromagnetics Research B, vol. 41, no. 2, pp. 251-268, 2012.

[17] M. Yuan and Y. Lin, "Model selection and estimation in regression with grouped variables," J. Royal Statist. Soc. Series B, vol. 68, no. 1, pp. 49 67, 2006.

[18] J. Huang and T. Zhang, "The benefit of group sparsity," Ann. Statist., vol. 38, no. 4, pp. 1978-2004, 2010. 